\title{
Quilotórax em linfoma não-Hodgkin: um relato de caso
}

\author{
Chylothorax in non-Hodgkin's lymphoma: a case report
}

Quilotóraxenel linfoma no Hodgkin: un informe de caso

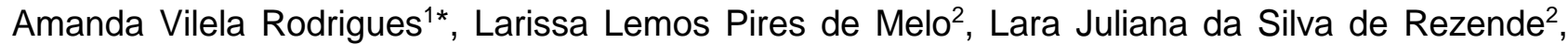
Lays Cristina Oliveira Campos², Eduardo Vilela Rodrigues ${ }^{1}$, Lyssa Ferreira', Ciro de Castro Botto'.
\end{abstract}

\section{RESUMO}

Objetivo: Descrever principais características do quilotórax relacionado ao linfoma não Hodgkin através de um relato. Detalhamento do caso: Trata-se de um relato de uma paciente do sexo feminino, já com diagnóstico de Linfoma não Hodgkin Folicular, admitida com sintomas de desnutrição, perda ponderal e dispnéia, demonstrando opacidade pulmonar homogênea até terço médio bilateral e obliteração de ambos os seios costofrênicos por derrame pleural do tipo quilotórax. Sendo o ducto torácico responsável por levar a linfa rica em nutrientes, gorduras e linfócitos para circulação venosa e estando este lesado o paciente cursa com desnutrição, emagrecimento e alteração na imunidade, algumas das queixas que levaram a paciente em questão a procurar por atendimento médico. Esta paciente respondeu a medidas clínicas não invasivas, não sendo necessário condutas cirúrgicas, porém existem diversas intervenções cirúrgicas para o tratamento do quilotórax. O tratamento clínico é preferível em relação ao tratamento cirúrgico por apresentar maior tempo de hospitalização, morbidade e mortalidade. Considerações finais: As publicações levantadas reunidas ao caso relatado descrevem as principais características relacionadas ao quilotórax e o Linfoma não Hodgkin, uma vez que este é um tipo de neoplasia não traumática que frequentemente gera a fistulização do ducto torácico e, como resultado, ocasiona o quilotórax.

Palavras-chave: Quilotórax, Linfoma não hodgkin, Linfoma folicular.

\begin{abstract}
Objective: To describe the main characteristics of chylothorax related to non-Hodgkin's lymphoma through a case report. Case Report: This is a case report of a female patient, already diagnosed with follicular nonHodgkin's lymphoma, admitted with symptoms of malnutrition, weight loss and dyspnea, showing homogeneous pulmonary opacity to bilateral middle third and obliteration of both costophrenic sinuses due to chylothorax-type pleural effusion. As the thoracic duct is responsible for taking lymph rich in nutrients, fats and lymphocytes to venous circulation and, being injured, the patient is suffering from malnutrition, weight loss and changes in immunity, some of the complaints that led the patient in question to seek medical attention. This patient responded to non-invasive clinical measures, without the need for surgical procedures, but there are several interventions for the treatment of chylothorax. Clinical treatment is preferable over surgical treatment because it has a longer hospital stay, morbidity and mortality. Final considerations: The publications raised in the reported case describe the main characteristics related to chylothorax and nonHodgkin's lymphoma, since this is a type of non-traumatic neoplasm that often generates fistulization of the thoracic duct and, as a result, causes chylothorax.
\end{abstract}

Key words: Chylothorax, Non-hodgkin's lymphoma, Follicular lymphoma.

\footnotetext{
${ }^{1}$ Fundação Santa Casa de Misericórdia de Franca, Franca-SP. *E-mail: mandrodiigues@outlook.com

2 Faculdade de Medicina de Franca (UNIFRAN), Franca-SP.
} 
RESUMEN

Objetivo: Describir las principales características del quilotórax relacionadas con el linfoma no Hodgkin a través de un informe de caso. Caso clínico: Este es un informe de caso de una paciente, ya diagnosticada con linfoma no Hodgkin folicular nodular, admitida con síntomas de desnutrición, pérdida de peso y disnea, que muestra opacidad pulmonar homogénea al tercio medio bilateral y obliteración de ambos senos costofrénicos debido al derrame pleural de tipo quilotórax. Como el conducto torácico es responsable de llevar la linfa rica en nutrientes, grasas y linfocitos a la circulación venosa y, al estar lesionado, el paciente sufre de desnutrición, pérdida de peso y cambios en la inmunidad, algunas de las quejas que llevaron al paciente en cuestión a buscar atención médica. Este paciente respondió a medidas clínicas no invasivas, sin la necesidad de procedimientos quirúrgicos, pero hay varias intervenciones para el tratamiento del quilotórax. El tratamiento clínico es preferible al tratamiento quirúrgico porque tiene una estancia hospitalaria más larga, morbilidad y mortalidad. Consideraciones finales: las publicaciones planteadas en el caso informado describen las características principales relacionadas con el quilotórax y el linfoma no Hodgkin, ya que este es un tipo de neoplasia no traumática que a menudo genera fistulización del conducto torácico y, como resultado, causa quilotórax.

Palabras clave: Quilotórax, Linfoma no Hodgkin, Linfoma folicular.

\section{INTRODUÇÃO}

Quilotórax é um tipo de derrame pleural raro, definido por acúmulo de quilo no espaço pleural. Este termo quilo é referente a aparência leitosa do líquido pleural rico em gordura, substância produzida no sistema linfático presente na região gastrointestinal (MECHÁN JMV, et al, 2011; MAIA I, et al., 2013). É causado por dano ao ducto torácico traumático ou não traumático, onde a ruptura traumática ocorre após acidentes ou cirurgias, e causas não traumáticas podem ser obstrução, compressão ou infiltração do ducto torácico, sendo o Linfoma não Hodgkin a causa mais prevalente entre elas (MECHÁN JMV, et al., 2011).

O quadro clínico é principalmente respiratório e cursa com desconforto, dessa forma a principal queixa é a dispneia, variando em sua gravidade com o volume do derrame pleural. O paciente também poderá ter 0 sistema imunológico prejudicado e apresentar-se emagrecido, desnutrido e desidratado devido à perda de gorduras, vitaminas lipossolúveis, proteínas, eletrólitos e linfócitos-T contidos na linfa (LIMA RJ, et al., 2009).

O diagnóstico do quilotórax é realizado após imagem sugestiva de derrame pleural com toracocentese apresentando líquido de aspecto leitoso associado a análise do líquido pleural com contagem de triglicerídeos com níveis acima de $110 \mathrm{mg} / \mathrm{dL}$, níveis inferiores a $50 \mathrm{mg} / \mathrm{dL}$ excluem essa patologia (MECHÁN JMV, et al., 2011; BABURAJ P, et al., 2016). Em níveis intermediários a estes valores é necessária análise das lipoproteínas do líquido pleural para a detecção de quilomícrons, que é considerado o padrão ouro no diagnóstico desse tipo de derrame pleural (VAZ MA, et al, 2006).

Pode ser realizado também linfografia de ducto torácico que demonstra o local de lesão do ducto torácico, e principalmente na identificação de más-formações linfáticas e linfocintilografia pode ser útil na identificação de ascite quilosa e na confirmação de quilotórax, principalmente naqueles pacientes onde há dúvida sobre o diagnóstico (VAZ MA, et al, 2006; LIMA RJ, et al., 2009).

O tratamento é preferencialmente conservador com toracocentese e drenagem em selo d'água para expansão pulmonar associados a dieta hipogordurosa ou dieta parenteral total livre de lipídeos com jejum oral (PORCEL JM, et al., 2019; KUMAR A, et al., 2014). É importante ressaltar que se há doença subjacente é necessário o tratamento adjunto (PORCEL JM, et al., 2019; KUMAR A, et al., 2014). Se o tratamento conservador falhar pode-se recorrer ao tratamento cirúrgico (MECHÁN JMV, et al., 2011).

Assim, este estudo possui o intuito de descrever as principais características do quilotórax relacionado ao linfoma não Hodgkin através de um relato de caso. 


\section{RELATO DE CASO}

Paciente sexo feminino, branca, 65 anos. Linfoma não Hodgkin Folicular diagnosticado há três meses após análise de anatomopatológico de implante secundário em omento após obstrução intestinal, ileostomia terminal e hipertensão arterial sistêmica. Em uso de Losartana e em tratamento quimioterápico. Procurou atendimento médico com relato de perda de mais de dez quilos, $20 \%$ do peso corporal total, com alimentação preservada e dispneia aos moderados esforços há dois meses, referindo piora importante na última semana, evoluindo para dispneia aos pequenos esforços, Escala Medical Research Council (MRC) grau 4 e ortopneia.

Ao exame físico na admissão apresentava regular estado geral, desnutrida, hipocorada 2+/4+, desidratada +/4+, anictérica, acianótica, taquipneica e taquicárdica. Escala de Glasgow 15. Aparelho Respiratório: murmúrio vesicular (MV) fisiológico abolido, percussão maciça e frêmito tóraco-vocal diminuído em bases e terço médio à direita e em uso de musculatura intercostal. Saturando $92 \%$ com cateter de oxigênio a 2L por minuto. Frequência respiratória: 27 incursões por minuto. Aparelho Cardiovascular: Bulhas rítmicas em 2 tempos, sem sopros. Frequência Cardíaca: 110 batimentos por minuto, Pressão Arterial: $110 \times 60 \mathrm{mmHg}$. Aparelho Gastrointestinal: Flácido, normotenso, sem dor ao palpar, ruídos hidroaéreos presentes, sem visceromegalias ou massas, ileostomia funcionante à direita. Principalmente, pela queixa respiratória foi então, realizado radiografia de tórax na admissão com opacidade homogênea até terço médio bilateral e obliteração de ambos seios costofrênicos (Figura 1).

Figura 1 - Radiografia de tórax póstero-anteriorevidencia opacidade homogênea até terço médio bilateral, com obliteração dos seios costofrênicos.

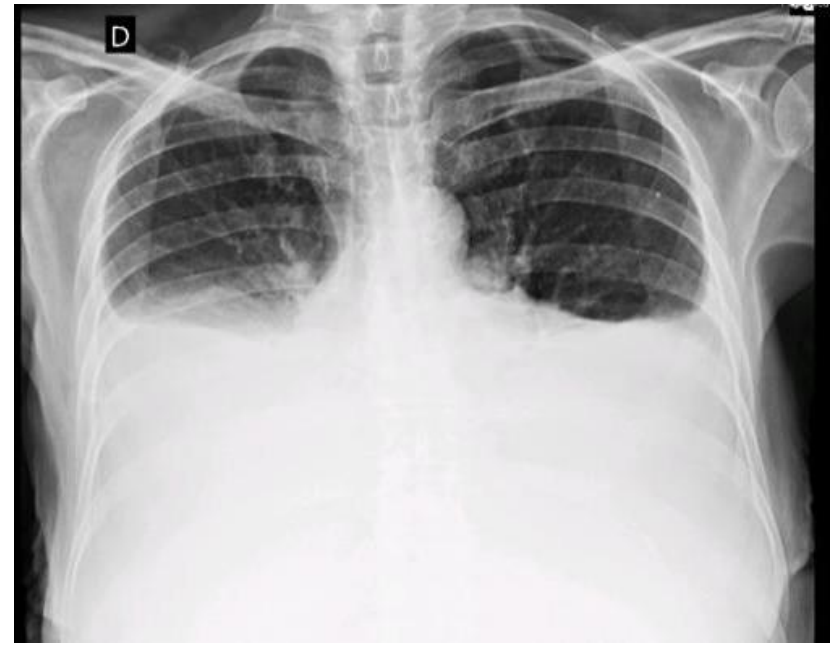

Fonte: Imagem cedida pelo setor de radiologia do hospital. Rodrigues AV, et al., 2019.

Após ser admitida na enfermaria da pneumologia foi realizado procedimento de toracocentese diagnóstica e alívio, à direita, com drenagem de $1040 \mathrm{~mL}$ de líquido pleural de aspecto leitoso: Descrição do líquido pleural: turvo, amarelo, pH7,7, leucócitos 20, Hemácias 45, Glicose 115, Proteínas 2,6, albumina 1,9, DHL 152, amilase 24 - não foi observado bactérias. Triglicerídeos no líquido pleural: 350mg/dL. Realizado drenagem de tórax em selo d'água sendo realizado 48horas após, com saída de $1200 \mathrm{~mL}$ de líquido pleural leitoso à direita e coletado biópsia da pleura com resultado negativo para neoplasia. Treze dias após a admissão, após ser confirmado o derrame pleural do tipo quilotórax foi alterado a dieta oral para dieta hipogordurosa.

O débito no dreno em selo d'água à direita de líquido pleural variou de 200 a 1000ml/dia com alteração do aspecto leitoso para coloração amarelo escuro três dias após a mudança na dieta para hipogordurosa. Mesmo com dieta hipogordurosa por dez dias, a drenagem de líquido pleural alternava o aspecto do líquido pleural com amarelo claro, escuro e leitoso, e manteve débito positivo 0 a $400 \mathrm{ml} /$ dia, sendo então optado por introduzir dieta parenteral sem lipídeos e jejum via oral. Ao exame físico respiratório apresentava 
murmúrio vesicular abolido em base com percussão maciça e frêmito tóraco-vocal diminuído à esquerda. Murmúrio vesicular fisiológico em hemitórax direito, sem ruídos adventícios. Frequência respiratória: 22irpm, Saturando $92 \%$ com cateter $2 \mathrm{~L}$, com uso de musculatura intercostal. Realizado novos exames de imagem: radiografia e tomografia computadorizada do tórax: opacidade homogênea até terço superior esquerdo gerando desvio do mediastino para direita, presença de dreno de tórax à direita, pulmão direito com boa expansibilidade (Figura 2, Figura 3).

Figura 2 - Tomografia computadorizada de tórax com derrame pleural a esquerda de grande volume.

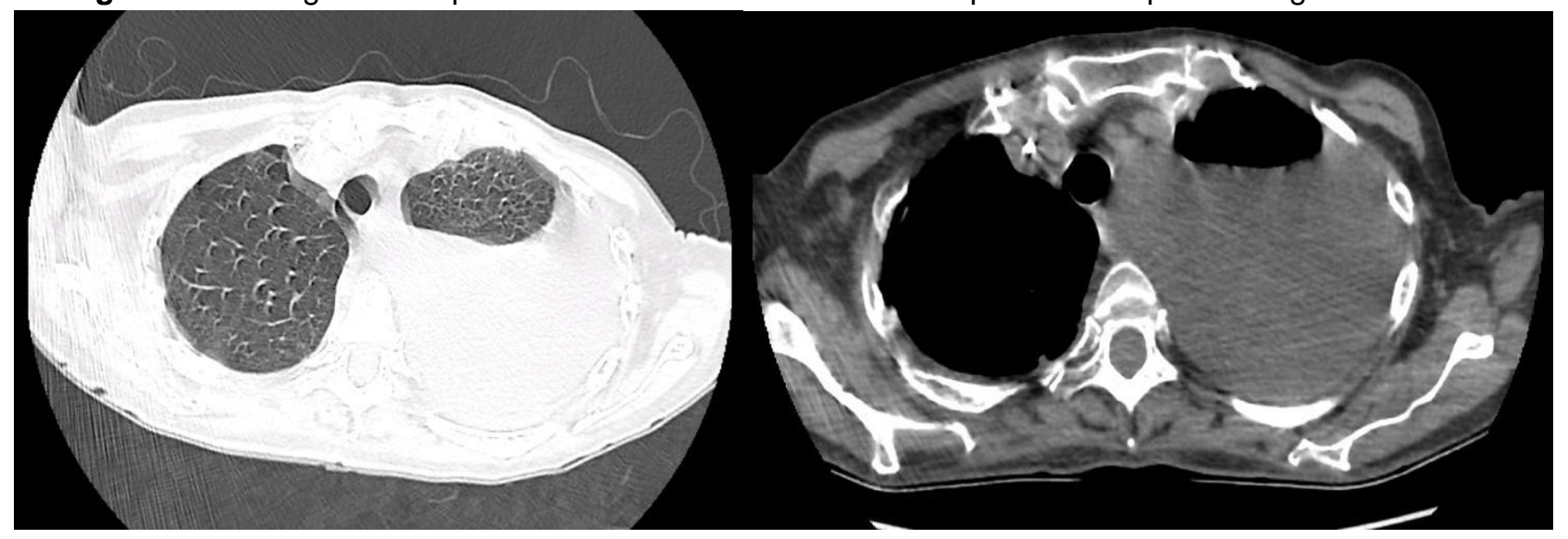

Fonte: Imagem cedida pelo setor de radiologia do hospital. Rodrigues AV, et al., 2019.

Figura 3 - Tomografia computadorizada de tórax com derrame pleural à esquerda, volumoso, pulmão direito com boa expansibilidade, presença de dreno de tórax, áreas de infiltrado em vidro fosco e nódulo pulmonar com densidade de partes moles, sub pleural em lobo inferior direito.

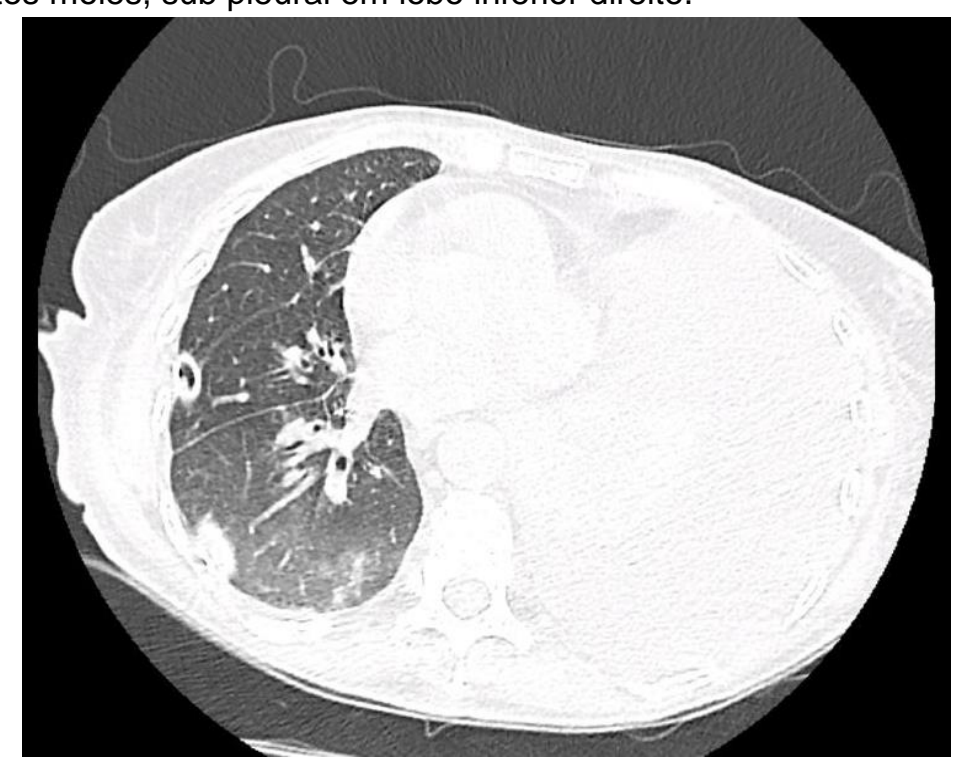

Fonte: Imagem cedida pelo setor de radiologia do hospital. Rodrigues AV, et al., 2019.

Sendo então realizado toracocentese de alívio em hemitórax esquerdo com drenagem de $1000 \mathrm{~mL}$ de líquido pleural de aspecto leitoso. Paciente manteve desconforto respiratório, sendo realizado 24horas após outra toracocentese de alívio à esquerda com mais $1500 \mathrm{~mL}$ de líquido drenado no mesmo aspecto.

Após duas toracocenteses à esquerda, o exame físico do Aparelho respiratório apresentava MV fisiológico abolido em base, com percussão submaciça e frêmito tóraco-vocal diminuído à esquerda. MV fisiológico à direita, sem ruídos adventícios. Sem uso de musculatura acessória. Saturando $86 \%$ em ar ambiente. Frequência Respiratória: 19irpm. 
Oito dias após a última toracocentese de alívio, a paciente apresentou novamente aumento da frequência e desconforto respiratório, sendo então realizado a terceira toracocentese à esquerda com drenagem de $1000 \mathrm{~mL}$ de líquido pleural desta vez com aspecto amarelo citrino. $O$ dreno de tórax à direita apresentava débito de $400 \mathrm{a} 800 \mathrm{~mL}$ de líquido variando o aspecto de amarelo-claro à seroso.

Foi reintroduzido a dieta com composição hiperlipídica para avaliar fechamento da fístula do ducto torácico 20 dias após ter sido instalado a nutrição parenteral sem lipídeos e jejum. Paciente apresentou 1 pico febril no $38^{\circ}$ de internação hospitalar sendo coletado hemoculturas, urocultura, cultura do cateter venoso central que foram negativas, e cultura do líquido pleural à esquerda que apresentou crescimento de Staphylococcus epidermidis, sendo iniciado antibiótico Vancomicina guiado pela cultura.

No período o dreno à direita apresentou líquido pleural amarelo citrino e posteriormente débito negativo, sendo retirado. Manteve ao exame físico, murmúrio vesicular fisiológico abolido em base e terço médio à esquerda, sendo realizado drenagem em selo d'água à esquerda no $41^{\circ}$ de internação hospitalar, mantendo drenagem de 0 a $400 \mathrm{~mL} /$ dia variando o aspecto de coloração amarelo escuro e citrino por cinco dias, sendo então retirado o dreno à esquerda.

Paciente recebeu alta em bom estado geral, dieta via oral para hipertenso, encaminhamento ambulatorial na oncologia para completar o tratamento da quimioterapia do Linfoma não Hodgkin Folicular Nodular. Atualmente encontra-se em bom estado geral, em recuperação da nutrição com ganho de peso e sem queixas respiratórias.

\section{DISCUSSÃO}

O quilotórax é um tipo importante de derrame pleural formado por quilo, sendo uma mistura da linfa absorvida no intestino de composição gordurosa e aspecto leitoso, tem sua maioria levada até a circulação venosa por meio do ducto torácico. A linfa proveniente da parede abdominal, fígado, pulmão e extremidades inferiores é drenada para a cisterna do quilo e levada ao ducto torácico (MECHÁN JMV, et al, 2011; JOHNSON OW, et al, 2016).

Quando ocorre obstrução do ducto torácico, seu acúmulo entre as pleuras causa sintomas respiratórios como os descritos no caso relatado, entre eles, principalmente, a dispneia aos pequenos e médios esforços com piora progressiva. É composto principalmente por gorduras, ácidos graxos livres, lecitina, esfingomielina, fosfolípedes e colesterol. A glicose varia de $40-200 \mathrm{mg} / \mathrm{dL}$ e a composição eletrolítica é similar a do plasma, contém vitaminas, anticorpos, enzimas como lipase, fosfatase alcalina, transaminases e ureia (LIMA RJ, et al., 2009). O quilotórax ocorre após a formação de fístula linfática por ruptura de vasos tributários distendidos e sua etiologia é dividida em traumática e não traumática (MAIA I, et al., 2013). Entre as principais causas das lesões não traumáticas estão os Linfomas do tipo não Hodgkin.

O local de acúmulo do quilo depende do local de acometimento no ducto torácico, por exemplo, se lesionado acima da quinta vértebra o quilotórax se apresenta bilateralmente (MECHÁN JMV, et al, 2011), como no caso do paciente referido. A apresentação clínica é arrastada, evolui à medida que ocorre o aumento do volume do derrame pleural causando efeitos mecânicos como dificuldade na expansão pulmonar cursando com dispneia progressiva e taquipneia (MECHÁN JMV, et al, 2011; BABURAJ P, et al., 2016). Sendo o ducto torácico responsável por levar a linfa rica em nutrientes, gorduras e linfócitos para circulação venosa e estando este lesado o paciente cursa com desnutrição, emagrecimento e alteração na imunidade (LIMA RJ, et al., 2009), algumas das queixas que levaram o paciente em questão a procurar por atendimento médico.

O diagnóstico do quilotórax é realizado de forma simples. Após a realização de imagem como radiografia ou tomografia do tórax solicitado pela sugestiva clínica do paciente é realizado toracocentese de alívio ou diagnóstica onde se apresenta um líquido pleural de aspecto leitoso.

Diante da coloração e aspecto do líquido pleural são levantadas hipóteses sendo solicitado a contagem de triglicerídeos no líquido pleural e sendo altamente sugestivo quando o resultado é superior a $110 \mathrm{mg} / \mathrm{dL}$ e exclui esta hipótese com valor inferior a 50mg/dL (MECHÁN JMV, et al, 2011). 
Um diagnóstico diferencial interessante é o pseudoquilotórax que se apresenta em aspecto leitoso e possui triglicerídeos inferior a $110 \mathrm{mg} / \mathrm{dL}$ com ausência de quilomícrons, sua etiologia pode ser em espessamento de pleuras, derrames pleurais crônicos, derrame pleural tuberculoso ou doenças reumatológicas. Ou o empiema pleural que também pode apresentar-se turvo a inspeção (VAZ MA, et al, 2006).

A presença de quilomícrons no líquido pleural detectados por eletroforese de lipoproteínas é considerado o padrão ouro no diagnóstico do quilotórax. Porém, sendo a contagem dos triglicerídeos no líquido pleural um método simples e barato opta-se por realizar este recurso (LIMA RJ, et al., 2009; RODRIGUES ALL, et al 2016).

O paciente referido foi submetido à toracocentese de alívio e diagnóstica, sendo que a análise do líquido pleural revelou $350 \mathrm{mg} / \mathrm{dL}$ de triglicerídeos, confirmando assim, a hipótese de quilotórax. Outros exames mais elaborados podem ser realizados em caso de dúvida diagnóstica, tais como linfografia de ducto torácico ou linfocintilografia (VAZ MA, et al, 2006; SCHILD HH, et al, 2013)

Após o diagnóstico, destaca-se a importância do tratamento da doença adjacente, capaz de resolver o quadro de quilotórax (PORCEL JM, et al., 2019; KUMAR A, et al., 2014). Porém, se o paciente se apresentar muito sintomático ou se a resolução da patologia principal não for possível é indicado instituir medidas terapêuticas como toracocentese de alívio ou drenagem em selo d'água. A dieta hipogordurosa é fundamental e há indicação de nutrição parenteral com dieta oral zero se o débito no dreno for superior a $1000 \mathrm{~mL} /$ dia ou se houver comprometimento do trato gastrointestinal (MAIA I, et al., 2013; CIRILO, MAS, et al, 2018).

A literatura fala sobre o uso da somatostatina e análogos que possuem ação bloqueadora das secreções gástricas, biliar, pancreáticas e intestinal sendo esperada também redução do fluxo no ducto torácico sendo indicada antes de indicar procedimentos invasivos (MECHÁN JMV, et al, 2011). Além disso, a tetraciclina ou a doxiciclina apresentam efeitos esclerosantes, os quais são capazes de reduzir o fluxo quiloso. (JUNIOR FMC, et al, 2009)

A conduta perante o paciente foi inicialmente toracocentese de alívio com dieta hipogordurosa pela via oral, porém logo depois se optou por drenagem em selo d'água para monitorização do débito e aspecto do líquido pleural diante das medidas terapêuticas. Sem resposta significativa foi prescrito dieta via oral zero e instalado nutrição parenteral. Após vinte dias em dieta oral zero e nutrição parenteral o líquido pleural apresentava-se de aspecto citrino drenado em selo d'água sendo então iniciada dieta hiperlipídica e observado o aspecto do líquido pleural que se manteve em coloração amarelo-claro confirmando o fechamento do ducto torácico podendo ser retirado as medidas de tratamento como drenagem em selo d'água e restrição de dieta.

Este paciente respondeu a medidas clínicas não invasivas, não sendo necessário condutas cirúrgicas, porém existem diversas intervenções para o tratamento do quilotórax que estão indicadas em média após quatorze dias de terapêutica clínica a depender do estado nutricional e imunológico do paciente que não obteve resposta positiva do tratamento conservador (ARSHAVA EV, 2019). São elas: Ligadura do ducto torácico, sutura da fístula do ducto torácico, pleurodese, pleurectomia, shunt-pleuroperitoneal, entre outras (MONTEIRO NP, et al, 2004). O tratamento clínico é preferível em relação ao tratamento cirúrgico pois a abordagem cirúrgica apresenta maior tempo de hospitalização, morbidade e mortalidade (MECHÁN JMV, et al, 2011).

\section{CONSIDERAÇÕES FINAIS}

As publicações levantadas reunidas ao caso relatado descrevem as principais características relacionadas ao quilotórax e as neoplasias tipo Linfoma não Hodgkin, uma vez que este tipo de neoplasia é uma forma não traumática que frequentemente gera a fistulização do ducto torácico e, como resultado, ocasiona o quilotórax. Desta forma, é primordial que diante de um paciente com derrame pleural do tipo quilotórax confirmado seja abordada a hipótese diagnóstica adicional de linfoma, e vice-versa, a fim de melhorar o diagnóstico das duas patologias em questão. 


\section{REFERÊNCIAS}

1. BABURAJ P, et al. Pleural Lymphoma Presenting as Chylothorax. Journal of The Association of Physicians of India, 2016; 64 (10), pp. 84-85.

2. CHEN HJ, et al. Diagnostic Pitfalls of Discriminating Lymphoma-Associated Effusions. Medicine, $2015 ; 94$ (17).

3. KUMAR A, et al. A 69-Year-Old Woman with Lymphoma and Chylothorax: Looking Beyond the Usual Suspect. Annals of the American Thoracic Society, 2014;11 (9).

4. LIMA RJ, et al. Quilotórax: A propósito de um caso clínico. Revista Portuguesa de Pneumologia, 2009; 15(3), pp. 521-527.

5. MAIA I, et al. Abordagem Nutricional no Doente com Quilotórax. Nutrícias, 2013, 19, pp. 32-34.

6. MOHAMED M, et al. Non-Hodgkin lymphoma manifesting as massive malignant. Internal Medicine Journal, $2015 ; 45$ (9), pp. 980-983.

7. PORCEL JM, et al. Pleural Effusions in Diffuse Large B-Cell Lymphoma: Clinical. Lung, 2019; $197(1)$, pp. 47-51.

8. VAZ MA, et al. Quilotórax. Jornal Brasileiro de Pneumologia, 2006, 32 (4), pp. 197-203.

9. MECHÁN JMV, et al. Quilotórax bilateral asociado a linfoma no Hodgkin, folicular. Génesis del quilotórax. Acta Médica Peruana, 2011, 28 (2), pp. 82-86.

10. CIRILO MAS, et al. Dieta enteral fonte em triglicerídeo de cadeia média como coadjuvante terapêutico em um caso de quilotórax bilateral secundário à linfoma folicular. Nutricíon Clínica y Dietetic Hospitalaria, 2018; 38(1), pp.170174.

11. JUNIOR FMC, et al. Nova abordagem terapêutica da fístula quilosa cervical grave por meio da embolização do ducto torácico. Revista Brasileira de Cirurgia Cabeça Pescoço, 2009 ; 38 (4), pp. 264 - 267.

12. JOHNSON OW, et al. The thoracic duct: clinical importance, anatomic variation, imaging, and embolization. European Radiology, 2016, 26, pp. 2482-2493.

13. ARSHAVA EV. Thoracic Duct and Chylothorax: General Considerations. lowa Thoracic Surgery, 2019.

14. SCHILD HH, et al. Treatment options in patients with chylothorax. Deutsches Arzteblatt International, 2013; 110(48), pp. 819-826.

15. MONTEIRO NP, et al. Derrame pleural quiloso na melorreostose: relato de caso com revis,,o da literature. Pulmão RJ, 2004; 13(4), pp. 282-285.

16. RODRIGUES ALL, et al. Quilotórax bilateral espontâneo após vômitos excessivos em criança. Revista Paulista de Pediatria, 2016; 34(4), pp. 518-521. 